\title{
Risk Analysis Based on AHP and Fuzzy Comprehensive Evaluation for Maglev Train Bogie
}

\author{
Chengxin Fan, Fengshan Dou, Baiming Tong, and Zhiqiang Long \\ Engineering Research Center of Maglev Technology, College of Mechatronics Engineering and Automation, \\ National University of Defense Technology, Changsha 410073, China
}

Correspondence should be addressed to Zhiqiang Long; lzq@maglev.cn

Received 15 September 2015; Revised 17 December 2015; Accepted 22 December 2015

Academic Editor: Jurgita Antucheviciene

Copyright (C) 2016 Chengxin Fan et al. This is an open access article distributed under the Creative Commons Attribution License, which permits unrestricted use, distribution, and reproduction in any medium, provided the original work is properly cited.

The maglev bogie is the key subsystem for maglev train security. To ensure life and property security, it is essential to evaluate its risk level before its operation. In this paper, a combinational method of analytic hierarchy process and fuzzy comprehensive evaluation is proposed to assess hazards in a complex maglev bogie system associated with multiple subsystems' failures. The very comprehensive identification of risk sources has been done by analyzing the structure of maglev bogie. Furthermore, based on the fuzzy theory, linguistic evaluation set is classified according to risk tolerance. The score of each risk factor is obtained by weighted sum of the result of fuzzy comprehensive evaluation. Our results show that the degree of maglev bogie's risk is within the range of acceptability. The merits of this work facilitate finding the weak links and determining the maintenance of maglev bogie system.

\section{Introduction}

Rail safety and reliability are highly related to national development, and the lives and property of people. As a new kind of railway vehicle, the maglev train faced the difficulties which form the performance analysis of safety due to lack of experiences on the long-term operations. Hence, the safety analysis should be carried out through the risk analysis, which is very different from that for the traditional train. Maglev train system includes a number of subsystems, where the bogie that has a significant impact on maglev train safety is an important part. In this paper, we focus on the risk analysis of low-medium speed maglev train bogie.

In recent years, several researchers have carried out investigations to gain a deep insight into the reliability evaluation of a bogie frame. Han et al. evaluated experimentally static strength for bogie frame of an urban maglev train including performing fatigue analysis, cumulative damage, and fatigue test based on a developed fatigue load and as a final outcome, aimed at proposing a fatigue strength evaluation method for the bogie frame of urban maglev [1]. Cui et al. corroborated the carrying capacity of a maglev train on the horizontal curve through the analysis of a single magnetic bogie to ensure reliability and safety [2]. However, the research about the risk analysis of low-medium speed maglev train bogie has been rarely done from a holistic point of view.

Since we realize that describing the reason of a bogie failure is a difficult issue because of comprehensive risk sources, for example, it is more complex to describe failures by a chain of events in a modeling process [1]. Furthermore, the probabilities of various basic events are difficult to collect and estimate. As a result, some common methods are unsuitable for the risk analysis of bogie system. In this case, it is necessary to propose a risk analysis method which is able to handle multiple factors and fuzziness combined with quantitative analysis. Hence, fuzzy set theory [3] is a useful tool to deal with imprecise and uncertain data. Meanwhile, analytic hierarchy process (AHP), proposed by Saaty [4], is a practical decision-making method $[5,6]$. The AHP could handle uncertain, imprecise, and subjective data and it also has robustness when solving practical ranking problems [7]. Fuzzy comprehensive evaluation (FCE) is a common method to analyze the risk of the environmental resources and construction of bridges, mines, and dams [8,9]. It is also used in the safety areas of public facilities and transportation [10, 11]. Song et al. corroborated that the triangular extent fuzzy AHP approach was more effective to evaluate self-ignition risks of coal piles than the trapezoidal fuzzy AHP method. 


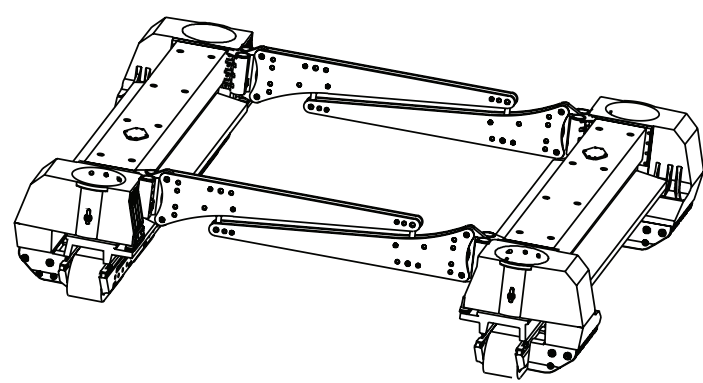

FIGURE 1: Bogie structure of low-medium speed maglev train.

They evaluated self-ignition risks of coal piles from a holistic point of view [12]. Yang et al. constructed a combination of AHP and FCE method to evaluate road transport system vulnerability against meteorological disasters from the view of risk analysis theory [13]. In our study, to overcome the complexity of structure and the uncertainty of various basic events' probabilities, a combinational method of AHP and FCE is proposed.

In Section 2, the maglev bogie structure and the relevant risk sources are analyzed. In Section 3, the three-layer hierarchical model is established to compute the weights distribution by using AHP. In Section 4, the risk degree of maglev bogie is assessed by using FCE. Finally, the discussion and concluding remarks are given in Sections 5 and 6.

\section{Risk Source Analysis of Maglev Bogie}

2.1. The Structure of a Maglev Bogie. Low-medium speed maglev train has five bogies. Air springs are installed on the four corners of each bogie. The bogies are connected with cars by 20 air springs, and the air suspension system consists of vertical and horizontal rod and steering mechanism. The bogies are not only the chassis of a train, but also the mounting platform of linear motors, solenoids, air springs, and other important power subassemblies. The bogies play an important role in delivering suspension force and steering force. Meanwhile, they also exert effect on geometric decoupling.

Figure 1 shows bogie structure of low-medium speed maglev train. A bogie consists of two left and two right modules which are connected together by two anti-roll beams. Each module consists of a linear motor beam, corbel, corbel connection, integrated bracket, transverse rod seat bracket, supporting slider, and hydraulic supporting wheel. The linear motor beam, corbel, and corbel connection are connected by bolts. The corbel connection and electromagnet are connected by keys and secured by bolts. The integrated bracket, corbel, and linear motor beam are connected together. The antiroll beam is installed outside the integrated bracket. The hydraulic supporting wheel is installed inside it. Antiroll beams are connected by suspenders. The linear motor is hanged by eight bolts in the lower part of linear motor beam [2].

2.2. Risk Source Analysis. According to the analysis of bogie structure, the main failures of bogie system and the reasons and consequences of these failures are as follows [14].
2.2.1. Corbel. The main reasons of corbel damage are foundry lacuna, fracture, losing suspension point, and split of wire rope seat. The corbel's damage and fracture may lead to air spring and electromagnet falling on track. Severe cases may cause train's damage and even casualties.

2.2.2. Corbel Connection. The faults of corbel connection are mainly due to the internal foundry lacuna and the damage of fatigue. The faults may lead to electromagnet's shedding. Severe cases may cause train's damage and even casualties.

2.2.3. Integrated Bracket. The main reasons of integrated bracket fracture are foundry lacuna and fatigue. This failure may lead to antiroll beam failing to connect with the left and right modules and disbanding of bogie structure. Severe cases may cause train's damage and even casualties.

2.2.4. Antiroll Beam. The causes of antiroll beam's fracture may be quality's defect and damage of knocking. If these faults happen, they will influence train's operation. They may cause train's damage and even casualties, especially when antiroll beam stuck between sleeper and train [15].

2.2.5. Linear Motor. The foundry lacuna and fatigue of linear motor beam can lead to the faults of linear motor. Snow and other obstructions on the track may lead to linear motor's burning out. The failures of linear motor may lead to losing power. Severe cases may cause train's damage and even casualties.

2.2.6. Electromagnet. The link package of electromagnet may have overcurrent faults. The outer plates of electromagnet and inner plates of electromagnet may be collided with obstacles. The failures of electromagnet may lead to critical consequences, such as abnormal suspension, train's smoking, and outbreak of a fire.

2.2.7. Air Spring. The main reasons of air spring's burst may be external force and aging. The failures of air spring may lead to train's abnormal operation, and the train may slow down or be rescued back to a maintenance location.

2.2.8. Mechanical Brake. The main reason of mechanical brake failure is screws' loosening of the outer plate of electromagnet. When the train carries out air brake, mechanical brake failure may lead to insufficient braking force. If the air brake and electric brake are both ineffective in extreme cases, the train will only execute brake by falling on the track. Therefore, normal train's operations will be interrupted [16].

The most of risk sources described above are foundry lacuna and abrasion of mechanical parts. These risk sources all can lead to the failures of maglev bogie. As a result, there are multiple initiating events. It is more complex to describe failures by a chain of events in a modeling process [1]. Furthermore, the probabilities of various basic events are difficult to collect and estimate. Hence, some common risk analysis techniques, for example, event tree analysis (ETA) and fault tree analysis (FTA), are not suitable for the risk analysis of bogie system. However, AHP and its extensions 
were developed to solve alternative selection criteria and justification issues that are capable of capturing a human's appraisal of ambiguity when complex multicriteria decisionmaking problems are considered $[9,17]$. FCE is an effective method which can use the experiences of engineers and analysts to express the uncertain relations of risk analysis [18]. It can give the level of risk for this system qualitatively. Therefore, this paper employs AHP and FCE to analyze the risk for maglev bogie system.

\section{Establishment of Analytic Hierarchy Process for Maglev Bogie}

3.1. Establishment of the Level Hierarchical Model. According to the analysis of the structure of maglev bogie, the eight sources of maglev bogie risk have been found, because there are a few subsystems' devices installed on the maglev bogie. These devices' faults can reduce different size risks. In order to analyze the contribution degree for total risk from each subsystem, it is essential to classify risk sources in accordance with the subsystems. The subsystems include the supporting unit, drive unit, architecture, brake unit, and suspension unit. The fifth risk source includes linear motor and linear motor beam. The linear motor and linear motor beam are part of drive unit. Meanwhile, the linear motor beam is also part of the architecture of maglev bogie. The sixth risk source includes line package, outer plates of electromagnet, and inner plates of electromagnet. These three factors are part of suspension unit. Because mechanical braking device is installed on the outer plate of electromagnet, the outer plate of electromagnet can affect brake unit at the same time. Therefore, there are 11 factors included in the five subsystems. The relationship between the 11 factors and the five subsystems are as follows:

(i) The support unit is a component or structure on the bogie which provides support for the quadratic suspension system. The structure of support unit includes air spring and corbel, and the air spring is installed in the corbel.

(ii) The drive unit is a component or structure which is used to drive maglev train. Linear motor and linear motor beam are part of drive unit. The linear motor is installed on the linear motor beam.

(iii) The architecture is the main mechanical structure of maglev bogie. It includes linear motors beam, corbel, integrated bracket, antiroll beam, and corbel connection. The integrated bracket is installed on the corbel. The antiroll beam is connected with the integrated bracket. The corbel connection is installed on the corbel.

(iv) The brake unit is the component which is installed on the bogie for braking. Mechanical braking device, outer plate of electromagnet, and corbel connection are part of brake unit. Mechanical braking device is installed on the outer plate of electromagnet. The outer plate of electromagnet is connected with the main structure of bogie by corbel connection.

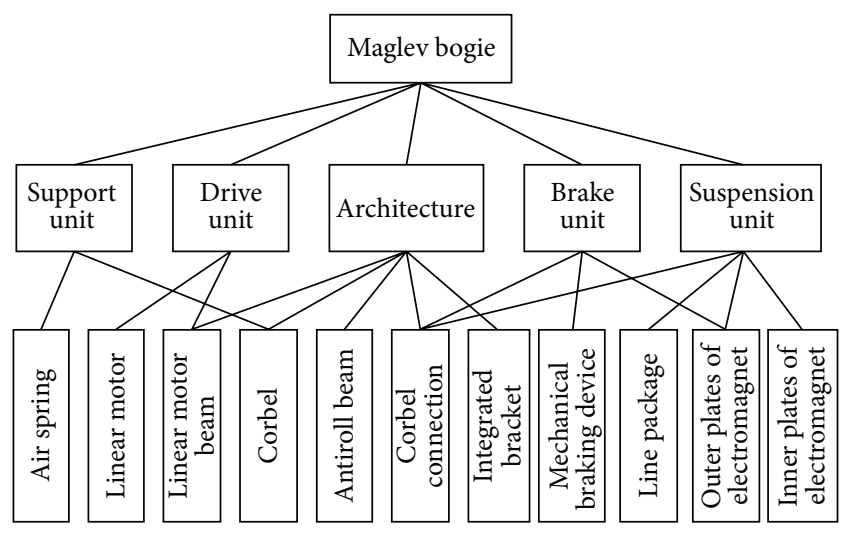

FIGURE 2: Level hierarchical model of bogie.

(v) The suspension unit provides vertical suspension force for maglev train. Link package, outer plates of electromagnet, inner plates of electromagnet, and corbel connection are part of the suspension unit. The suspension electromagnet line package and core are installed between the inner plates of electromagnet and outer plates of electromagnet. The inner plates of electromagnet and outer plates of electromagnet are connected with the main structure of bogie by corbel connections.

Accordingly, a three-layer hierarchical model is established to describe maglev bogie reasonably $[19,20]$. The target layer is the risk of maglev bogie. The first layer (criterion layer) is the subsystems of maglev bogie, which includes the supporting unit, drive unit, architecture, brake unit, and suspension unit. The second layer (scheme layer) includes air spring, linear motor, linear motor beam, corbel, antiroll beam, corbel connection, integrated bracket, mechanical braking device, line package, outer plates of electromagnet, and inner plates of electromagnet.

The level hierarchical model of bogie is shown in Figure 2.

To describe the hierarchical model more clearly, we give the corresponding notations in Table 1.

The set of factors can be represented as

$$
\mathbf{U}=\left\{u_{i 1}^{(l)}, u_{i 2}^{(l)}, \ldots, u_{i n_{i}}^{(l)}\right\},
$$

where $u_{i n_{i}}^{(l)}$ is the $n$ factor of the $l$ layer dominated by the $i$ factor of the $l-1$ layer.

The weight vector of factors is represented as

$$
\begin{aligned}
& \mathbf{W}=\left\{w_{1}^{(l)}, w_{2}^{(l)}, \ldots, w_{n}^{(l)}\right\}, \\
& \mathbf{A}=\left\{a_{1}^{(l)}, a_{2}^{(l)}, \ldots, a_{n}^{(l)}\right\},
\end{aligned}
$$

where $\mathbf{W}$ is the weight which is determined by normalizing the eigenvector and $\mathbf{A}$ is the weight vector of factors.

\subsection{Calculation of Factors' Weights under a Single Criterion}

3.2.1. Determination of the Weights of the Second Layer Factor Set with respect to the First Layer Criterion Set. It is assumed that the factor $u_{i}^{(l-1)}$ of the $l-1$ layer is a criterion. The next 
TABLE 1: Factor set partitions and symbolic representations of bogie's level hierarchical mode.

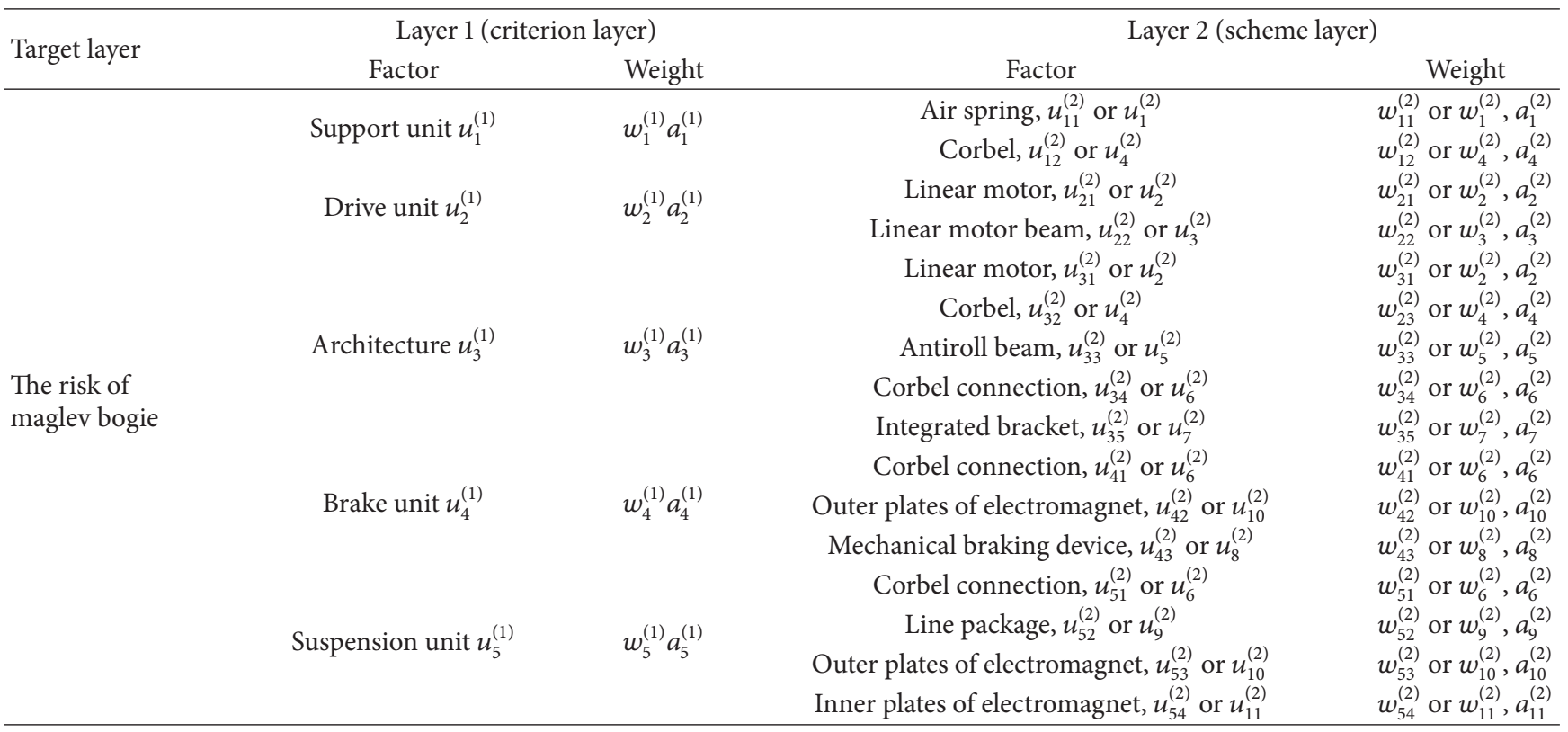

layer factors $u_{i 1}^{(l)}, u_{i 2}^{(l)}, \ldots, u_{i n_{i}}^{(l)}$ are dominated by the criterion. The judgment matrix is determined by comparing relative importance between two arbitrary factors from $u_{i 1}^{(l)}, u_{i 2}^{(l)}, \ldots, u_{i n_{i}}^{(l)}[4]$.

In this study, the judgment matrix is determined by expert decision along with field investigation and other related research results [16], given as follows:

$$
\mathbf{C}_{1}^{(1)}=\left[\begin{array}{cc}
1 & 5 \\
\frac{1}{5} & 1
\end{array}\right]
$$

where $\mathbf{C}_{1}^{(1)}$ is the judgment matrix of the first layer's first factor.

The maximum eigenvalue, $\lambda_{1, \max }^{(1)}=2$, is determined by $\mathbf{C}_{1}^{(1)}$.

The eigenvector can be calculated by

$$
\mathbf{C}_{i}^{(l-1)} w_{i}^{\prime(l-1)}=\lambda_{i, \max }^{(l-1)} w_{i}^{(l-1)},
$$

where $\mathbf{C}_{i}^{(l-1)}$ the judgment matrix, $\lambda_{i, \max }^{(l-1)}$ is the maximum eigenvalue, and $w_{i}^{\prime(l-1)}$ is the eigenvector [4].

$w_{1}^{(1)}$ is the weight which is determined by normalizing the eigenvector:

$$
w_{1}^{(1)}=\left[w_{11}^{(2)}, w_{12}^{(2)}\right]^{T}=[0.8333,0.1667]^{T} .
$$

It is essential to check the consistency of the judgment matrix to ensure that the calculated weights are acceptable. The consistency ratio can be determined by

$$
\begin{gathered}
\text { C.I. }_{1}^{(1)}=\frac{\lambda_{1, \max }^{(1)}-n_{1}^{(l)}}{n_{1}^{(l)}-1}=0, \\
\text { C.R }{ }_{1}^{(1)}=\frac{\text { C.I. }_{1}^{(1)}}{\text { R.I. }_{1}^{(1)}}=0<0.1,
\end{gathered}
$$

where C.I. ${ }_{1}^{(1)}$ is the consistency index, R.I. ${ }_{1}^{(1)}$ is the random index, and C.R. ${ }_{1}^{(1)}$ is the consistency ratio (C.R.). Saaty has
TABLE 2: The random consistency index (R.I.).

\begin{tabular}{lccccccccc}
\hline Size & 1 & 2 & 3 & 4 & 5 & 6 & 7 & 8 & 9 \\
\hline R.I. & 0 & 0 & 0.52 & 0.89 & 1.12 & 1.26 & 1.36 & 1.41 & 1.46 \\
\hline
\end{tabular}

proposed a consistency index (CI) and calculated the random indices shown in Table 2 [4].

The consistency of the judgment matrix is acceptable only if C.R. $<0.1$. Hence, $\mathbf{C}_{1}^{(1)}$ satisfies consistency requirement.

Similarly, based on the AHP which is introduced by Saaty [4], the other four judgment matrixes and their consistency are given as

$$
\begin{aligned}
\mathbf{C}_{2}^{(1)} & =\left[\begin{array}{ll}
1 & 5 \\
\frac{1}{5} & 1
\end{array}\right], \\
\lambda_{2, \max }^{(1)} & =2, \\
w_{2}^{(1)} & =\left[w_{21}^{(2)}, w_{22}^{(2)}\right]^{T}=[0.8333,0.1667]^{T}, \\
\mathbf{C . I}_{2}^{(1)} & =\frac{\lambda_{2, \max }^{(1)}-n_{2}^{(1)}}{n_{2}^{(1)}-1}=0, \\
\text { C.R.R }{ }_{2}^{(1)} & =\frac{\text { C.I. } ._{2}^{(1)}}{\text { R.I. }} \cdot 2
\end{aligned}
$$

Hence, $\mathbf{C}_{2}^{(1)}$ satisfies consistency requirement. Consider

$$
\mathrm{C}_{3}^{(1)}=\left[\begin{array}{ccccc}
1 & 2 & \frac{1}{5} & \frac{1}{2} & \frac{1}{5} \\
\frac{1}{2} & 1 & \frac{1}{3} & \frac{1}{5} & \frac{1}{5} \\
5 & 3 & 1 & 1 & 1 \\
2 & 5 & 1 & 1 & 1 \\
5 & 5 & 1 & 1 & 1
\end{array}\right]
$$




$$
\begin{aligned}
\lambda_{3, \max }^{(1)} & =5.1703, \\
w_{3}^{(1)} & =\left[w_{31}^{(2)}, w_{32}^{(2)}, w_{33}^{(2)}, w_{34}^{(2)}, w_{35}^{(2)}\right]^{T} \\
& =[0.0884,0.0605,0.2852,0.2573,0.3086]^{T}, \\
\text { C.I. }{ }_{3}^{(1)} & =\frac{\lambda_{3, \max }^{(1)}-n_{3}^{(1)}}{n_{3}^{(1)}-1}=0.0426, \\
\text { C.R }{ }_{3}^{(1)} & =\frac{\text { C.I. }{ }_{3}^{(1)}}{\text { R.I. }_{3}^{(1)}}=\frac{5.1703}{1.12}=0.0380<0.1 .
\end{aligned}
$$

Hence, $\mathbf{C}_{3}^{(1)}$ satisfies consistency requirement. Consider

$$
\begin{aligned}
\mathbf{C}_{4}^{(1)} & =\left[\begin{array}{lll}
1 & 3 & 7 \\
\frac{1}{3} & 1 & 5 \\
\frac{1}{7} & \frac{1}{5} & 1
\end{array}\right], \\
\lambda_{4, \max }^{(1)} & =3.0649, \\
w_{4}^{(1)} & =\left[w_{41}^{(2)}, w_{42}^{(2)}, w_{43}^{(2)}\right]^{T} \\
& =[0.6491,0.2790,0.0719]^{T}, \\
\text { C.I. }{ }_{4}^{(1)} & =\frac{\lambda_{4, \max }^{(1)}-n_{4}^{(1)}}{n_{4}^{(1)}-1}=0.0324, \\
\text { C.R. }{ }_{4}^{(1)} & =\frac{\mathbf{C . I}_{4}^{(1)}}{\mathbf{R . I}_{4}^{(1)}}=\frac{0.0324}{0.52}=0.0624<0.1 .
\end{aligned}
$$

Hence, $\mathbf{C}_{4}^{(1)}$ satisfies consistency requirement. Consider

$$
\begin{aligned}
\mathbf{C}_{5}^{(1)} & =\left[\begin{array}{llll}
1 & 1 & 5 & 7 \\
1 & 1 & 3 & 5 \\
\frac{1}{5} & \frac{1}{3} & 1 & 3 \\
\frac{1}{7} & \frac{1}{5} & \frac{1}{3} & 1
\end{array}\right], \\
\lambda_{4, \max }^{(1)} & =4.0820, \\
w_{5}^{(1)} & =\left[w_{51}^{(2)}, w_{52}^{(2)}, w_{53}^{(2)}, w_{54}^{(2)}\right]^{T} \\
& =[0.4532,0.3633,0.1253,0.0581]^{T}, \\
\text { C.I. }{ }_{5}^{(1)} & =\frac{\lambda_{5, \max }^{(1)}-n_{5}^{(1)}}{n_{5}^{(1)}-1}=0.0273, \\
\text { C.R. }{ }_{5}^{(1)} & =\frac{\mathbf{C . I}{ }_{5}^{(1)}}{\mathbf{R . I}\left({ }_{5}^{(1)}\right.}=\frac{0.0273}{0.89}=0.0307<0.1 .
\end{aligned}
$$

Hence, $\mathbf{C}_{5}^{(1)}$ satisfies consistency requirement.
3.2.2. Determination of the Weights of the First Layer Criterion Set with respect to the Target Set. The judgment matrix is given as follows:

$$
\mathbf{C}^{(0)}=\left[\begin{array}{ccccc}
1 & \frac{1}{3} & \frac{1}{5} & 3 & \frac{1}{6} \\
3 & 1 & \frac{1}{5} & 1 & \frac{1}{5} \\
5 & 5 & 1 & 6 & 1 \\
\frac{1}{3} & 1 & \frac{1}{6} & 1 & \frac{1}{5} \\
6 & 5 & 1 & 5 & 1
\end{array}\right] .
$$

The results of the maximum eigenvalue and weight vector are given as

$$
\begin{aligned}
\lambda_{\max }^{(0)} & =5.4135, \\
w^{(0)} & =\left[w_{1}^{(1)}, w_{2}^{(1)}, w_{3}^{(1)}, w_{4}^{(1)}, w_{5}^{(1)}\right]^{T} \\
& =[0.0805,0.1027,0.3762,0.0608,0.3798]^{T} .
\end{aligned}
$$

The consistency ratio can be determined by

$$
\begin{aligned}
& \text { C.I. }^{(0)}=\frac{\lambda_{\max }^{(0)}-n^{(0)}}{n^{(0)}-1}=0.1034, \\
& \text { C.R. }{ }^{(0)}=\frac{\text { C.I. }^{(0)}}{\text { R.I. }^{(0)}}=\frac{0.1034}{1.12}=0.0923<0.1 .
\end{aligned}
$$

Hence, $\mathbf{C}^{(0)}$ satisfies consistency requirement.

3.3. Calculation of Combining Weights of Each Layer Factor. The weight of the first layer factors with respect to the total target is $\mathbf{A}^{(1)}=w^{(0)}$. The single criterion layer weights of the second layer factors are as follows:

$$
\begin{aligned}
\bar{w}_{1}^{(1)} & =\left[w_{1}^{(2)}, w_{2}^{(2)}, \ldots, w_{n_{l}}^{(2)}\right]^{T} \\
& =\left[\begin{array}{lllllllllll}
0.8333 & 0 & 0 & 0.1667 & 0 & 0 & 0 & 0 & 0 & 0 & 0
\end{array}\right]^{T},
\end{aligned}
$$

where $\bar{w}_{1}^{(1)}$ is the weight vector of the first layer's first factor in a single order and $n_{l}$ is the number of factors in $l(l=2)$ layer. The factors dominated by an upper layer are nonzero:

$$
\begin{aligned}
& \bar{w}_{2}^{(1)}=\left[\begin{array}{lllllllllll}
0 & 0.8333 & 0.1667 & 0 & 0 & 0 & 0 & 0 & 0 & 0 & 0
\end{array}\right]^{T}, \\
& \bar{w}_{3}^{(1)} \\
& =\left[\begin{array}{lllllllllll}
0 & 0 & 0.0884 & 0.0605 & 0.2852 & 0.2573 & 0.3086 & 0 & 0 & 0 & 0
\end{array}\right]^{T}, \\
& \bar{w}_{4}^{(1)}=\left[\begin{array}{lllllllllll}
0 & 0 & 0 & 0 & 0 & 0.6491 & 0.2790 & 0.0719 & 0 & 0 & 0
\end{array}\right]^{T}, \\
& \bar{w}_{5}^{(1)}=\left[\begin{array}{lllllllllll}
0 & 0 & 0 & 0 & 0 & 0.4532 & 0 & 0 & 0.3633 & 0.1253 & 0.0581
\end{array}\right]^{T} \text {, } \\
& \mathbf{W}^{(2)}=\left[\begin{array}{llll}
\bar{w}_{1}^{(1)} & \bar{w}_{2}^{(1)} & \ldots & \bar{w}_{n_{l-1}}^{(1)}
\end{array}\right],
\end{aligned}
$$

where $\mathbf{W}^{(2)}$ is a $n_{l} \times n_{l-1}$ matrix and $n_{l-1}$ is the number of factors in $l-1(l=2)$ layer.

The weight vector of the second layer factors with respect to the total target in a combinational order can be calculated 
TABLE 3: Risk matrix.

\begin{tabular}{|c|c|c|c|c|c|}
\hline & \multicolumn{5}{|c|}{ Hazardous event severity level } \\
\hline & Negligible & Slight & Moderate & Serious & Disastrous \\
\hline \multicolumn{6}{|c|}{ Possibility of hazardous event } \\
\hline Frequently & Higher & Higher & Very high & Highest & Highest \\
\hline Very likely & Middle & High & Higher & Very high & Highest \\
\hline Occasionally & Lower & Middle & High & Very high & Very high \\
\hline Small possible & Very low & Lower & Middle & Higher & Very high \\
\hline Impossible & Very low & Very low & Low & High & Higher \\
\hline
\end{tabular}

by $\mathbf{A}^{(2)}=\mathbf{W}^{(2)} \mathbf{A}^{(1)}$. The results of vector $\mathbf{A}^{(2)}$ are $a_{1}^{(2)}=$ $0.0671, a_{2}^{(2)}=0.0856, a_{3}^{(2)}=0.0504, a_{4}^{(2)}=0.0362, a_{5}^{(2)}=$ $0.1073, a_{6}^{(2)}=0.3084, a_{7}^{(2)}=0.1331, a_{8}^{(2)}=0.0044, a_{9}^{(2)}=$ $0.1380, a_{10}^{(2)}=0.0476$, and $a_{11}^{(2)}=0.0221$.

The overall consistency of the weights of each layer factor with respect to the total target should be checked. For the first layer, because of $\mathbf{C} . \mathbf{R} .^{(1)}=\mathbf{C} . \mathbf{R} .^{(0)}$ it has a satisfactory overall consistency.

For the second layer,

$$
\begin{aligned}
& \text { C.I. }^{(2)}=\left[\begin{array}{lllll}
\text { C.I. }_{1}^{(1)} & \text { C.I. } ._{2}^{(1)} & \text { C.I. }{ }_{3}^{(1)} & \text { C.I. } ._{4}^{(1)} & \text { C.I. } ._{5}^{(1)}
\end{array}\right] \\
& \cdot \mathbf{A}^{(1)}=0.0284 \text {, }
\end{aligned}
$$

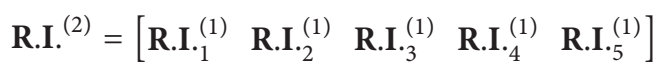

$$
\begin{aligned}
& \cdot \mathbf{A}^{(1)}=0.7910 \text {, } \\
& \text { C.R. }{ }^{(2)}=\frac{\text { C.I. }^{(2)}}{\text { R.I. }^{(2)}}=\frac{0.0284}{0.7910}=0.0359<0.1 \text {. }
\end{aligned}
$$

The overall consistency of the second layer satisfies the requirement.

According to calculations of this section, the weights of factors with respect to the total target are obtained.

\section{Risk Analysis Based on Fuzzy Comprehensive Evaluation for Maglev Bogie}

4.1. Division of Risk Level and Tolerance. Risk can be defined as follows [21]:

$$
\text { Risk }=\text { probability } \times \text { severity. }
$$

The mishap probability factor is the probability of the hazard components occurring and transforming into the mishap. The mishap severity factor is the overall consequence of the mishap.

The probability of fault maglev bogie occurring and the level of hazardous event severity are difficult to determine quantitatively. Therefore, linguistic terms [10] are applied to describe the probability and severity qualitatively. The probabilities of hazardous event are frequently, very likely, occasionally, small possible, and impossible. The hazardous event severity levels are disastrous, serious, moderate, slight, and negligible, respectively. The risks of maglev bogie are divided into eight levels, very low, lower, low, middle, high, higher, very high, and highest, respectively.
The risk matrix used to evaluate the level of risk for maglev bogie is shown in Table 3. It is determined by referencing IEC 62278: 2002 combined with engineering practice $[16,22]$.

Linguistic terms have been found intuitively easy to use in expressing the subjectiveness and imprecision of risk assessments $[3,23]$. In fact, the risk levels given by experts are fuzzy. Such an uncertain and imprecise issue can be expressed by the use of the fuzzy set theory [3].

In this paper the triangular fuzzy number is used for fuzzy numbers, because it is simpler than trapezoidal fuzzy number [24] and in accordance with the actual case. The definition of triangular fuzzy number is as follows, based on the operations on fuzzy numbers of Dubois and Prade [25]:

$$
\mu_{M}(x)= \begin{cases}\frac{1}{m-x} x-\frac{l}{m-l} & x \in[l, m], \\ \frac{1}{m-u} x-\frac{u}{m-u} & x \in[m, u], \\ 0 & \text { otherwise, }\end{cases}
$$

where $M$ is the fuzzy number, $\mu_{M}$ is the membership function of $M, m$ is called the most possible value of triangular fuzzy number $M$, and $l$ and $u$ are called lower and upper limits of $M$, respectively.

The eight linguistic terms of risk level are characterized by triangular fuzzy numbers for representing their approximate value range between 1 and 8 [26]. Figure 3 shows the memberships of these linguistic terms. The eight top points' membership functions are 1 , which represents the most possible eight linguistic terms of risk level, respectively. When each linguistic term's membership grade decreases, the other one will increase accordingly.

The risk levels which include very low, lower, and low are divided into acceptable risk. The middle, high, and higher risk levels are considered to be undesirable risk. The very high and highest risk levels are regarded as unacceptable. These rules are given according to the practical engineering experience. Through the analysis, three broken blue line triangles are drawn in Figure 4.

The $x$-coordinates of the crossover point of the two broken blue line triangles are 3.33 and 6.5 , respectively. The ranges of risk tolerance are obtained by comparing the size of memberships. They are given as follows:

(i) Acceptable risk: $[1,3.33)$.

(ii) Undesirable risk: $[3.33,6.5]$.

(iii) Unacceptable risk: $(6.5,8]$.

Figure 4 shows the division of risk tolerance. 


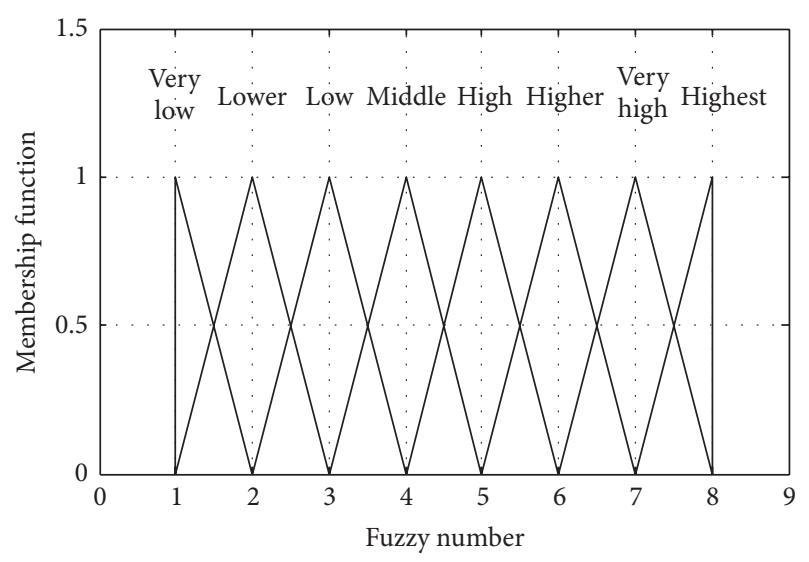

FIGURE 3: Membership functions of linguistic terms.

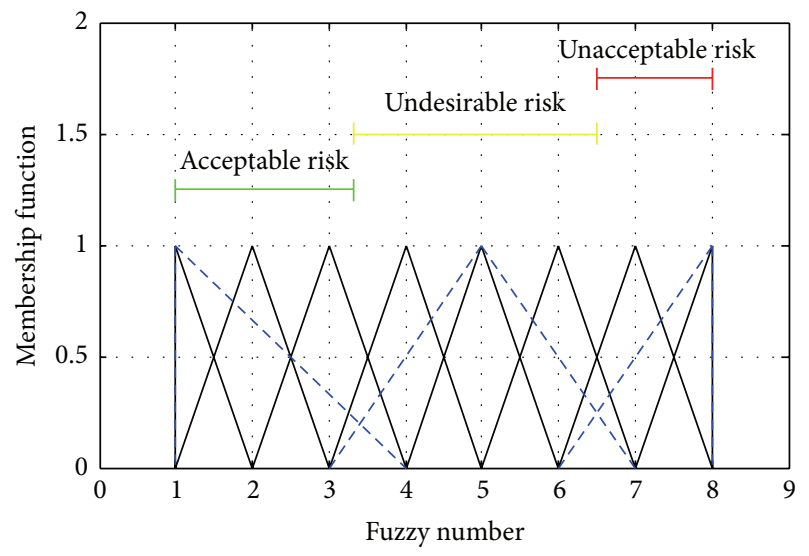

FIGURE 4: Division of risk tolerance.

4.2. Fuzzy Comprehensive Risk Assessment. The weights of each layer factor with respect to the total target are obtained by using AHP. The weights used in FCE are obtained by normalizing them.

For the first level FCE, the weight vectors of the second layer's factors with respect to the total target are as follows:

$$
\begin{aligned}
& \mathbf{A}_{1}^{(2)}=\left[\begin{array}{ll}
a_{1}^{(2)} & a_{4}^{(2)}
\end{array}\right]=\left[\begin{array}{ll}
0.0671 & 0.0362
\end{array}\right], \\
& \mathbf{A}_{2}^{(2)}=\left[\begin{array}{ll}
a_{2}^{(2)} & a_{3}^{(2)}
\end{array}\right]=\left[\begin{array}{ll}
0.0856 & 0.0504
\end{array}\right], \\
& \mathbf{A}_{3}^{(2)}=\left[\begin{array}{lllll}
a_{3}^{(2)} & a_{4}^{(2)} & a_{5}^{(2)} & a_{6}^{(2)} & a_{7}^{(2)}
\end{array}\right] \\
& =\left[\begin{array}{lllll}
0.0504 & 0.0362 & 0.1073 & 0.3084 & 0.1331
\end{array}\right], \\
& \mathbf{A}_{4}^{(2)}=\left[\begin{array}{lll}
a_{6}^{(2)} & a_{8}^{(2)} & a_{10}^{(2)}
\end{array}\right]=\left[\begin{array}{lll}
0.3084 & 0.0044 & 0.0476
\end{array}\right], \\
& \mathbf{A}_{5}^{(2)}=\left[\begin{array}{llll}
a_{6}^{(2)} & a_{9}^{(2)} & a_{10}^{(2)} & a_{11}^{(2)}
\end{array}\right] \\
& =\left[\begin{array}{llll}
0.3084 & 0.1380 & 0.0476 & 0.0221
\end{array}\right] \text {. }
\end{aligned}
$$

Before starting the first level FCE, the above-mentioned five weights should be normalized, given as

$$
\begin{aligned}
& \underline{\mathbf{A}}_{1}^{(2)}=\left[\begin{array}{ll}
0.6496 & 0.3504
\end{array}\right], \\
& \underline{\mathbf{A}}_{2}^{(2)}=\left[\begin{array}{ll}
0.6294 & 0.3706
\end{array}\right] \text {, } \\
& \underline{\mathbf{A}}_{3}^{(2)}=\left[\begin{array}{lllll}
0.0793 & 0.0570 & 0.1689 & 0.4854 & 0.2095
\end{array}\right] \text {, } \\
& \underline{\mathbf{A}}_{4}^{(2)}=\left[\begin{array}{lll}
0.8557 & 0.0122 & 0.1321
\end{array}\right], \\
& \stackrel{\mathbf{A}}{5}_{5}^{(2)}=\left[\begin{array}{llll}
0.5976 & 0.2674 & 0.0922 & 0.0428
\end{array}\right] .
\end{aligned}
$$

For the second level FCE, the weight of the first layer factor set is $\underline{\mathbf{A}}^{(1)}$, that is, $\mathbf{A}^{(1)}$.

According to Table 3, the evaluation set of FCE can be written as $\mathbf{V}=$ \{very low, lower, low, middle, high, higher, very high, highest\}. In order to make the index quantitative, the grade is provided for the corresponding evaluation set: $N=(1,2,3,4,5,6,7,8)[5]$.

When the first level FCE starts, risk analysts give the single-factor evaluation matrix $\mathbf{R}_{i}^{(2)}$ of the second layer. This can be calculated by using model $M(\cdot,+)$ to begin fuzzy compositional operation “o". $\mathbf{R}_{i}^{(2)}$ is determined by [27]

$$
\mathbf{R}_{i}^{(2)}=\left[\begin{array}{cccc}
r_{i 11}^{(2)} & r_{i 12}^{(2)} & \cdots & r_{i 1 m}^{(2)} \\
r_{i 21}^{(2)} & r_{i 22}^{(2)} & \cdots & r_{i 2 m}^{(2)} \\
\vdots & \vdots & & \vdots \\
r_{i n_{i}^{(2)} 1}^{(2)} & r_{i n_{i}^{(2)} 2}^{(2)} & \cdots & r_{i n_{i}^{(2)} m}^{(2)}
\end{array}\right] \quad i=1,2, \ldots, n^{(1)}
$$

where $n^{(1)}\left(n^{(1)}=5\right)$ represents the number of factors included in the first layer, $n_{i}^{(2)}$ denotes the number of factors included in the second layer with respect to the factor $i$ of the first layer, and $m(m=8)$ stands for the number of the evaluative sets.

Hence, the matrix $\mathbf{R}_{1}^{(2)}$ can be derived as follows:

$$
\mathbf{R}_{1}^{(2)}=\left[\begin{array}{cccccccc}
0.1 & 0.2 & 0.3 & 0.3 & 0.06 & 0.04 & 0 & 0 \\
0.2 & 0.3 & 0.3 & 0.1 & 0.1 & 0 & 0 & 0
\end{array}\right]
$$

Similarly, the matrices $\mathbf{R}_{2}^{(2)}, \mathbf{R}_{3}^{(2)}, \mathbf{R}_{4}^{(2)}$, and $\mathbf{R}_{5}^{(2)}$ are obtained. They are shown as follows:

$$
\begin{aligned}
\mathbf{R}_{2}^{(2)} & =\left[\begin{array}{llllllll}
0.06 & 0.2 & 0.2 & 0.3 & 0.2 & 0.04 & 0 & 0 \\
0.05 & 0.3 & 0.3 & 0.3 & 0.05 & 0 & 0 & 0
\end{array}\right], \\
\mathbf{R}_{3}^{(2)} & =\left[\begin{array}{ccccccccc}
0.05 & 0.3 & 0.3 & 0.3 & 0.05 & 0 & 0 & 0 \\
0.2 & 0.3 & 0.3 & 0.1 & 0.1 & 0 & 0 & 0 \\
0.04 & 0.25 & 0.25 & 0.25 & 0.2 & 0.01 & 0 & 0 \\
0.1 & 0.3 & 0.3 & 0.2 & 0.1 & 0 & 0 & 0 \\
0.1 & 0.2 & 0.3 & 0.3 & 0.1 & 0 & 0 & 0
\end{array}\right],
\end{aligned}
$$




$$
\begin{aligned}
\mathbf{R}_{4}^{(2)} & =\left[\begin{array}{cccccccc}
0.1 & 0.3 & 0.3 & 0.2 & 0.1 & 0 & 0 & 0 \\
0.05 & 0.1 & 0.2 & 0.2 & 0.3 & 0.1 & 0.05 & 0 \\
0.1 & 0.1 & 0.2 & 0.3 & 0.2 & 0.1 & 0 & 0
\end{array}\right], \\
\mathbf{R}_{5}^{(2)} & =\left[\begin{array}{cccccccc}
0.1 & 0.3 & 0.3 & 0.2 & 0.1 & 0 & 0 & 0 \\
0.05 & 0.18 & 0.36 & 0.3 & 0.1 & 0.01 & 0 & 0 \\
0.1 & 0.1 & 0.2 & 0.3 & 0.2 & 0.1 & 0 & 0 \\
0.1 & 0.2 & 0.3 & 0.25 & 0.15 & 0 & 0 & 0
\end{array}\right] .
\end{aligned}
$$

The results of evaluation can be obtained through multiplying the vector of the factor weight and the matrix $\mathbf{R}$ of single-factor evaluation [5]:

$$
\begin{aligned}
& \stackrel{\mathbf{B}}{\sim}_{1}^{(2)}={\underset{\mathbf{A}}{1}}_{1}^{(2)} \circ \mathbf{R}_{1}^{(2)} \\
& =\left[\begin{array}{llllllll}
0.1350 & 0.2350 & 0.3000 & 0.2299 & 0.0740 & 0.0260 & 0 & 0
\end{array}\right],
\end{aligned}
$$

where ${\underset{\sim}{\mathbf{B}}}_{1}^{(2)}$ is the evaluation result of the first layer's first factor. The elements in $\mathbf{B}_{1}^{(2)}$ are membership of the evaluation object with regard to the elements in the evaluation set. The conclusion of the comprehensive evaluation can be obtained by the maximum membership principle [5].

Similarly, the results of the first level FCE are given, respectively, by

$$
\begin{aligned}
& \underline{\sim}_{2}^{(2)}={\underset{\sim}{2}}_{2}^{(2)} \circ \mathbf{R}_{2}^{(2)} \\
& =\left[\begin{array}{llllllll}
0.0563 & 0.2371 & 0.2371 & 0.3000 & 0.1444 & 0.0252 & 0 & 0
\end{array}\right], \\
& \underline{\mathbf{B}}_{3}^{(2)}={\underset{\mathbf{A}}{3}}_{3}^{(2)} \circ \mathbf{R}_{3}^{(2)} \\
& =\left[\begin{array}{llllllll}
0.0916 & 0.2706 & 0.2916 & 0.2316 & 0.1129 & 0.0017 & 0 & 0
\end{array}\right], \\
& {\underset{\sim}{(}}_{4}^{(2)}={\underset{\mathbf{A}}{4}}_{4}^{(2)} \circ \mathbf{R}_{4}^{(2)} \\
& =\left[\begin{array}{llllllll}
0.0994 & 0.2711 & 0.2856 & 0.2132 & 0.1157 & 0.0144 & 0.0006 & 0
\end{array}\right] \text {, } \\
& {\underset{\sim}{5}}_{5}^{(2)}=\underline{\mathbf{A}}_{5}^{(2)} \circ \mathbf{R}_{5}^{(2)} \\
& =\left[\begin{array}{llllllll}
0.0866 & 0.2452 & 0.3068 & 0.2381 & 0.1114 & 0.0119 & 0 & 0
\end{array}\right] \text {. }
\end{aligned}
$$

The results of the second level FCE are given by

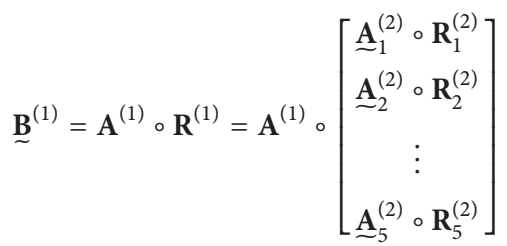

$$
\begin{aligned}
& =\left[\begin{array}{llllllll}
0.0900 & 0.2547 & 0.2921 & 0.2399 & 0.1126 & 0.0107 & 0 & 0
\end{array}\right],
\end{aligned}
$$

where ${\underset{\sim}{\mathbf{B}}}^{(1)}$ is the fuzzy comprehensive evaluation result of risk level for maglev bogie.

The maximum membership of ${\underset{\mathbf{B}}{(1)}}^{(1)}$ is the three-factor 0.2921 which indicates the total risk level of maglev bogie is low. The maximum membership of $\underset{\sim}{\mathbf{B}_{1}^{(2)}}, \underset{\sim}{\mathbf{B}_{2}^{(2)}}, \underset{\sim}{\mathbf{B}_{3}^{(2)}}, \underset{\sim}{\mathbf{B}_{4}^{(2)}}$, and ${\underset{\mathbf{B}}{5}}_{5}^{(2)}$ are $0.3,0.3,0.2916,0.2856$, and 0.3068 , respectively. Similarly, the risk level of supporting unit, architecture, brake unit, and suspension unit is low. The risk level of drive unit is middle. Hence, maintainers should pay more attention to the drive unit.
4.3. Results of Risk Assessment. In order to get the risk tolerance of each factor, weighted sum of the result of risk level is proposed.

The index is determined by

$$
\text { Index }=\underset{\sim}{\mathbf{B}} \cdot \mathbf{N} \text {, }
$$

where Index is the score of risk evaluation and $\mathbf{N}(\mathbf{N}=$ $\left[\begin{array}{llllllll}1 & 2 & 3 & 4 & 5 & 6 & 7 & 8\end{array}\right]^{T}$ ) is the grade of evaluation set.

The single-factor evaluation matrix of scheme layer is corresponding to the row vector of $\mathbf{R}_{i}^{(2)}$, and $\mathbf{A}^{(3)}=1$.

Table 4 shows the risk assessment of bogie system determined by results of FCE.

According to Figure 3, the risk index of bogie system is acceptable in Table 4. The risks of five subsystems are also acceptable. However, there are four parts' risk indexes undesirable. They are linear motor, antiroll beam, mechanical braking device, and outer plates of electromagnet, respectively. Clearly, they are the weak links which affect the safety of bogie system. Accordingly, making decisions for the maintenance of maglev train is convenient. Meanwhile, the cost is dropped remarkably when putting the limited resources into the most needed place.

\section{Discussion}

The main difficulties in risk analysis for mechatronics systems are the complexity of mechanism and the uncertainty of various basic events' probabilities. The research about risk analysis for maglev bogie is very rare.

As a response to this dilemma, various methods have been proposed for solving risk analysis problems for mechatronics systems. For example, the frequently used methods are ETA and FTA. However, ETA is unable to handle the process of identifying safety risk because of multiple no related events. FTA can identify hazards that present mishap risk with an assessment of the risk [21]. But as the probabilities of various basic events are difficult to collect and estimate, FTA is unable to give quantitative risk assessment.

In this paper, the combinational method of analytic hierarchy process and fuzzy comprehensive evaluation has been proposed to solve the complex and uncertain problems in the area of risk analysis for maglev bogie. First, the complex structure of maglev bogie should be taken into consideration. There are a few subsystems' devices installed on the maglev bogie. These devices' faults can reduce different size risks. To deal with this challenge, based on the analysis of maglev bogie's structure, 11 risk sources have been divided into five groups in accordance with the subsystems to determine the contribution degree for total risk. Risk sources and risk sources' reasons and effects have influence on finding the relationship between failures and grading risks. In this study, the identification of risk sources has been very comprehensive. But the analysis of risk sources' reasons and effects is limited, and it is also the importance of future research.

Second, the application of AHP in this study is mainly based on Saaty's research [4]. The hierarchy relationships of risk factors have been built clearly by AHP. Certainly, AHP still suffers from some theoretical disputes. The assumption of 
TABLE 4: Results of risk assessment.

\begin{tabular}{|c|c|c|c|c|}
\hline Level & Item & Index & Risk & Ratio \\
\hline System & Bogie & 3.0627 & Acceptable & \\
\hline \multirow{5}{*}{ Subsystem } & Support unit & 2.9506 & Acceptable & 0.1919 \\
\hline & Drive unit & 3.3150 & Acceptable & 0.2156 \\
\hline & Architecture & 3.0090 & Acceptable & 0.1957 \\
\hline & Brake unit & 3.0203 & Acceptable & 0.1965 \\
\hline & Suspension unit & 3.0782 & Acceptable & 0.2002 \\
\hline \multirow{11}{*}{ Component } & Air spring & 3.1400 & Acceptable & 0.0877 \\
\hline & Corbel & 2.6000 & Acceptable & 0.0726 \\
\hline & Linear motor & 3.5000 & Undesirable & 0.0978 \\
\hline & Linear motor beam & 3.0000 & Acceptable & 0.0838 \\
\hline & Antiroll beam & 3.3500 & Undesirable & 0.0936 \\
\hline & Corbel connection & 2.9000 & Acceptable & 0.0810 \\
\hline & Integrated rated bracket & 3.1000 & Acceptable & 0.0866 \\
\hline & Mechanical braking device & 4.1000 & Undesirable & 0.1146 \\
\hline & Line package & 3.2500 & Acceptable & 0.0908 \\
\hline & Outer plates of electromagnet & 3.7000 & Undesirable & 0.1034 \\
\hline & Inner plates of electromagnet & 3.1500 & Acceptable & 0.0880 \\
\hline
\end{tabular}

criteria independence may be sometimes a limitation of AHP [6]. The selection of judgment matrix is completed by experts' engineering practice experience. In fact, exact numbers are inappropriate for linguistic judgments. Hence, fuzzy AHP [28] may be considered in the next research.

Third, the conclusion of maglev bogie's risk level obtained by the maximum membership principle [5] is low. However, each factor's risk performance should be considered into risk analysis to find the weak links of maglev bogie. Based on the fuzzy theory [3], linguistic evaluation set has been classified according to risk tolerance. The fuzzy comprehensive evaluation method is utilized to assess the score and ratio of each factor. Accordingly, it is convenient for people to make decisions for the maintenance of maglev bogie system.

\section{Conclusion}

In this paper, we have proposed an effective risk analysis method, a combinational method of analytic hierarchy process and fuzzy comprehensive evaluation, which provides a quantitative risk assessment for low-medium speed maglev train.

The very comprehensive identification of risk sources has been done by analyzing the structure of maglev bogie. The weight of each layer is calculated by analytic hierarchy process. The results of risk level for maglev bogie are obtained by fuzzy comprehensive evaluation, and based on the maximum membership principle, the total risk level of maglev bogie is low. As the risk level of drive unit is middle, maintainers should pay more attention to the drive unit.

The triangular fuzzy number has been used to determine the ranges of risk tolerance. The score of each risk factor is obtained by weighted sum of the result of fuzzy comprehensive evaluation. The results of risk assessment show that the risk degree of maglev bogie is acceptable. However, the risk degrees of linear motor, antiroll beam, mechanical braking device, and outer plates of electromagnet are undesirable. Clearly, they are the weak links which affect the safety of bogie system.

The evaluated results can provide a reasonable guide to analyze the risk of maglev bogie system. The merits of the approach facilitate finding the weak links and making decisions for the maintenance of maglev bogie system. The costs will also decrease significantly when putting the limited resources into the most needed place.

\section{Conflict of Interests}

The authors declare that there is no conflict of interests regarding the publication of this paper.

\section{Acknowledgment}

This work is supported by the National Key Technology R\&D Program of the 12th Five-Year Plan, Systematic Study on Engineering Integration of High Speed Maglev Transportation, 2013BAG19B01.

\section{References}

[1] J.-W. Han, J.-D. Kim, and S.-Y. Song, "Fatigue strength evaluation of a bogie frame for urban maglev train with fatigue test on full-scale test rig," Engineering Failure Analysis, vol. 31, pp. 412-420, 2013.

[2] P. Cui, J. Li, and D. S. Liu, "Carrying capacity for the electromagnetic suspension low-speed maglev train on the horizontal curve," Science China Technological Sciences, vol. 53, no. 4, pp. 1082-1087, 2010.

[3] L. A. Zadeh, "Fuzzy sets," Information and Computation, vol. 8, pp. 338-353, 1965.

[4] T. L. Saaty, "A scaling method for priorities in hierarchical structures," Journal of Mathematical Psychology, vol. 15, no. 3, pp. 234-281, 1977. 
[5] J.-F. Chen, H.-N. Hsieh, and Q. H. Do, "Evaluating teaching performance based on fuzzy AHP and comprehensive evaluation approach," Applied Soft Computing Journal, vol. 28, pp. 100-108, 2015.

[6] A. Ishizaka and A. Labib, "Review of the main developments in the analytic hierarchy process," Expert Systems with Applications, vol. 38, no. 11, pp. 14336-14345, 2011.

[7] B. Srdjevic and Y. D. P. Medeiros, "Fuzzy AHP assessment of water management plans," Water Resources Management, vol. 22, no. 7, pp. 877-894, 2008.

[8] Q. Nian, S. Shi, and R. Li, "Research and application of safety assessment method of gas explosion accident in coal mine based on GRA-ANP-FCE," Procedia Engineering, vol. 45, pp. 106-111, 2012.

[9] S. Su, X. Chen, S. D. DeGloria, and J. Wu, "Integrative fuzzy set pair model for land ecological security assessment: a case study of Xiaolangdi Reservoir Region, China," Stochastic Environmental Research and Risk Assessment, vol. 24, no. 5, pp. 639-647, 2010.

[10] C.-H. Yeh, H. Deng, and Y.-H. Chang, "Fuzzy multicriteria analysis for performance evaluation of bus companies," European Journal of Operational Research, vol. 126, no. 3, pp. 459-473, 2000.

[11] A. Roozbahani, B. Zahraie, and M. Tabesh, "Integrated risk assessment of urban water supply systems from source to tap," Stochastic Environmental Research and Risk Assessment, vol. 27, no. 4, pp. 923-944, 2013.

[12] Z. Song, H. Zhu, G. Jia, and C. He, “Comprehensive evaluation on self-ignition risks of coal stockpiles using fuzzy AHP approaches," Journal of Loss Prevention in the Process Industries, vol. 32, no. 1, pp. 78-94, 2014.

[13] J. Yang, H. Sun, L. Wang, L. Li, and B. Wu, "Vulnerability evaluation of the highway transportation system against meteorological disasters," Procedia-Social and Behavioral Sciences, vol. 96, pp. 280-293, 2013.

[14] Z. Ren, Fatigue Analysis and Simulation of Maglev Bogie in LowMedium Speed Maglev Train, National University of Defense Technology, Changsha, China, 2005.

[15] C. Huang, W. Xu, S. Yao, and B. Zheng, "Low-medium speed maglev train bogie anti-roll beam structure optimization," Rolling Stock, vol. 51, 2013.

[16] B. Tong, Risk Analysis of the Critical Systems in MediumLow Speed Maglev Train and its Application Research, National University of Defense Technology, Changsha, China, 2014.

[17] J. K. Levy, "Multiple criteria decision making and decision support systems for flood risk management," Stochastic Environmental Research and Risk Assessment, vol. 19, no. 6, pp. 438-447, 2005.

[18] Y. Han, W. Zhou, and X. Zhang, "Fuzzy comprehensive evaluation of the adaptability of an expressway system," Journal of Highway and Transportation Research and Development, vol. 8, no. 4, pp. 97-103, 2014.

[19] H. Yuan and W. Cai, "The model combined the BT project and land development risk assessment research," Systems Engineering Procedia, vol. 1, pp. 250-256, 2011.

[20] E. Cagno, F. Caron, and A. Perego, "Multi-criteria assessment of the probability of winning in the competitive bidding process," International Journal of Project Management, vol. 19, no. 6, pp. 313-324, 2001.

[21] C. A. Ericson, Hazard Analysis Techniques for System Safety, John Wiley \& Sons, Hoboken, NJ, USA, 2005.
[22] International Electrotechnical Commission, IEC 62278: Railway Applications-Specification and Demonstration of Reliability, Availability, Maintainability and Safety (RAMS), IEC Central Office, Geneva, Switzerland, 2002.

[23] G.-S. Liang, "Fuzzy MCDM based on ideal and anti-ideal concepts," European Journal of Operational Research, vol. 112, no. 3, pp. 682-691, 1999.

[24] E. Triantaphyllou and L. Chi-Tun, "Development and evaluation of five fuzzy multiattribute decision-making methods," International Journal of Approximate Reasoning, vol. 14, no. 4, pp. 281-310, 1996.

[25] D. Dubois and H. Prade, "Operations on fuzzy numbers," International Journal of Systems Science, vol. 9, no. 6, pp. 613626, 1978.

[26] E. K. Zavadskas and J. Antucheviciene, "Multiple criteria evaluation of rural building's regeneration alternatives," Building and Environment, vol. 42, no. 1, pp. 436-451, 2007.

[27] X. Yihong and S. Mengju, "Fuzzy comprehensive evaluation of beverage enterprise risks from system engineering perspective," Systems Engineering Procedia, vol. 3, pp. 240-248, 2012.

[28] O. S. Vaidya and S. Kumar, "Analytic hierarchy process: an overview of applications," European Journal of Operational Research, vol. 169, no. 1, pp. 1-29, 2006. 


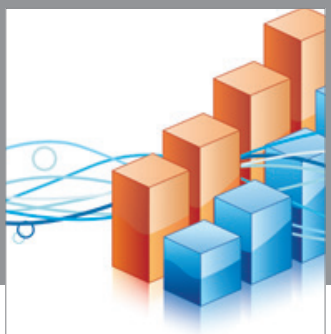

Advances in

Operations Research

vatem alat4

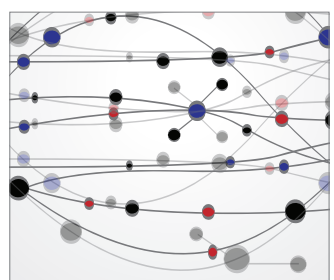

\section{The Scientific} World Journal
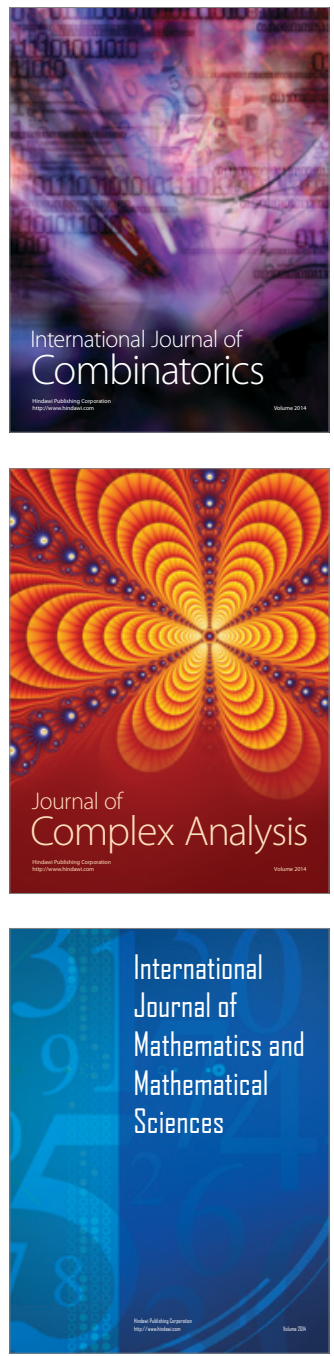
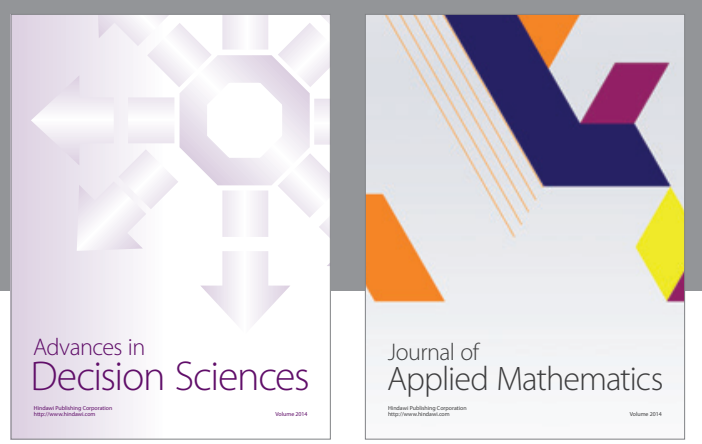

Algebra

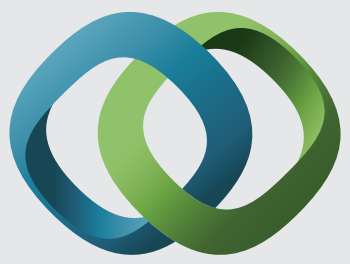

\section{Hindawi}

Submit your manuscripts at

http://www.hindawi.com
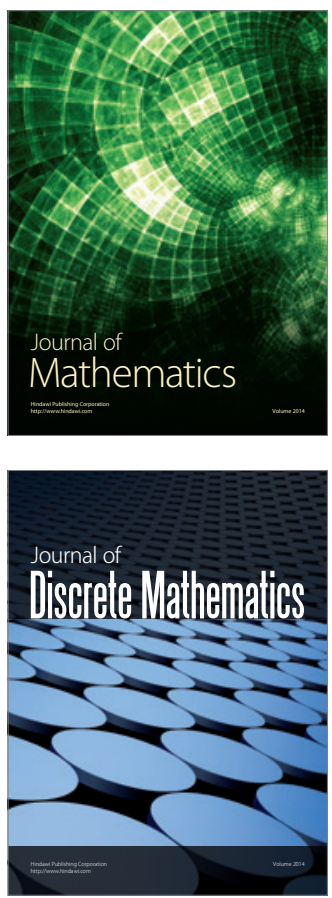

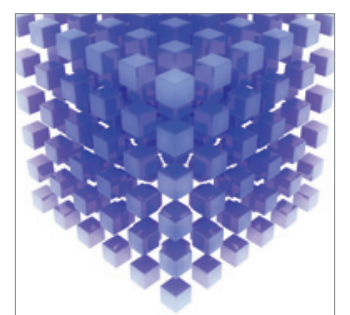

Mathematical Problems in Engineering
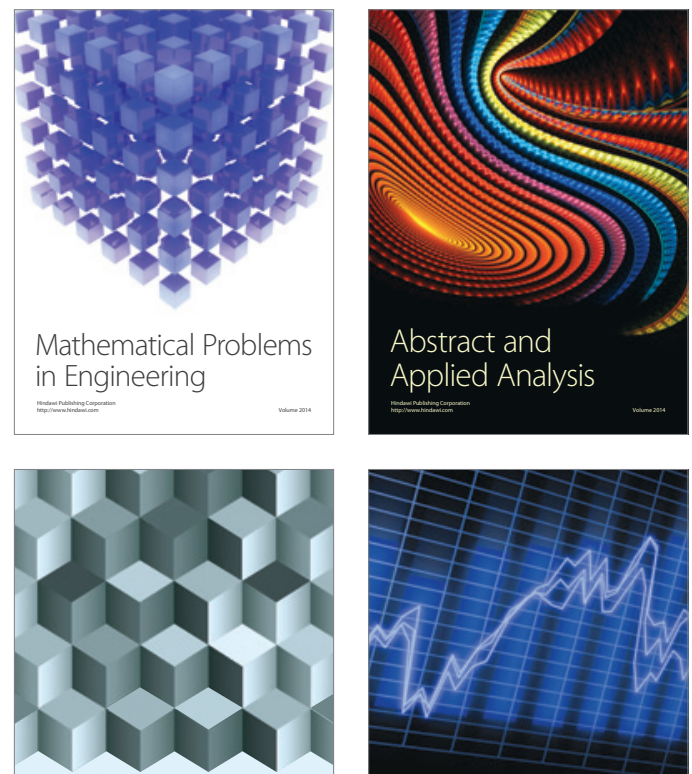

Journal of

Function Spaces

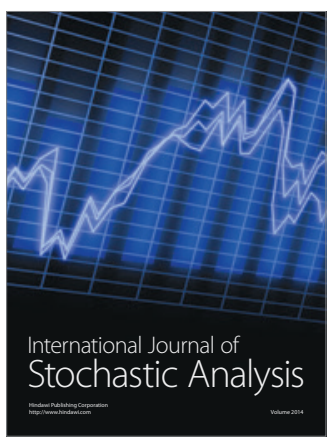

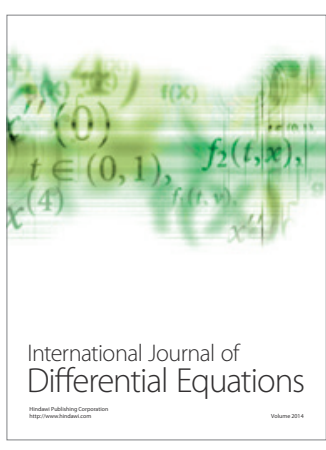
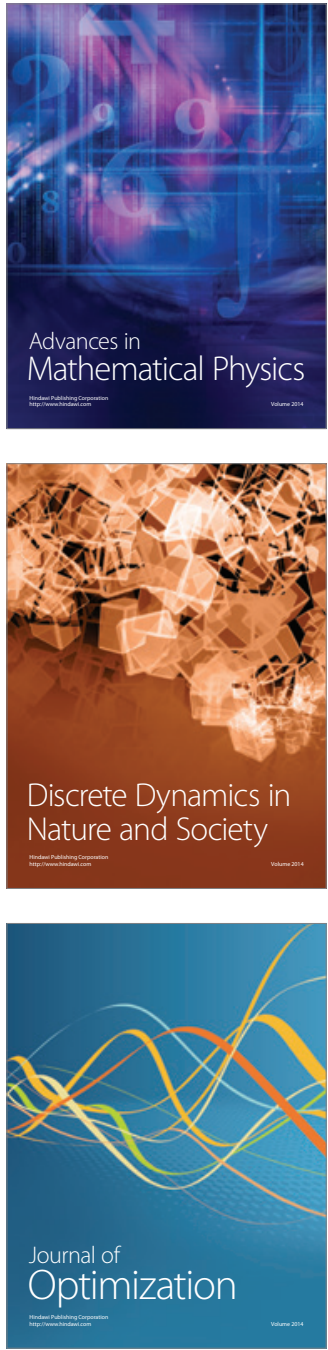